\title{
Bimetallic platinum-based complexes: syntheses and synchrotron X-ray radiation structural studies
}

\author{
I Yakushev ${ }^{1}$, I Stolarov ${ }^{1}$, M Vargaftik ${ }^{1}$ \\ ${ }^{1}$ Kurnakov Institute of general and inorganic chemistry (RAS), Moscow, Russian Federation \\ cs68@mail.ru
}

Today, the bimetallic palladium-based carboxylic complexes shown high reactivity in homogeneous catalytic hydrogenation processes of unsaturated hydrocarbons, and their effectiveness as precursors of supported heterogeneous bimetallic catalysts with improved activity and selectivity. Until recently, convenient singlemolecular precursors of the supported catalysts, platinum-based carboxylates were not available because of inaccessibility and low reactivity of the starting material platinum acetate $\left[\mathrm{Pt}_{4}(\mathrm{OOCMe})_{8}\right]$. In this work, we synthesized more than 20 novel homo- and heterometallic acetate complexes of platinum and some their derivatives with N-donor ligands. Two synthetic approaches were developed based on the platinum acetate blue (PAB, $\left.\mathrm{Pt}(\mathrm{OOCMe})_{2.25-2.75}\right)[1]$ and potassium hexahydroxyplatinate $\mathrm{K}_{2}\left[\mathrm{Pt}(\mathrm{OH})_{6}\right][2,3]$ as starting materials. The structures of the complexes were esrablished by conventional single-crystal X-ray diffraction technique and with using synchrotron radiation single crystal XRD methods. («Belok» beamline of the specialized source of synchrotron radiation "KISI-Kurchatov", National Research Center «Kurchatov Institute», Moscow, Russian Federation) [4]. Acknowledgments: This work is supported by the Russian Science Foundation under grant 18-73-10206. This research was performed using the equipment of the JRC PMR IGIC RAS. References: [1] N.V. Cherkashina, D.I. Kochubey, V.V. Kanazhevskiy, V.I. Zaikovskii, V.K. Ivanov, A.A. Markov, A P. Klyagina, Zh.V. Dobrokhotova, N.Y. Kozitsyna, I.B. Baranovsky, O.G. Ellert, N.N. Efimov, S.E. Nefedov, V.M. Novotortsev, M.N. Vargaftik, I.I. Moiseev. // Inorganic Chemistry, V. 53 (2014), 8397-8406. [2] Ilya A. Yakushev, Igor P. Stolarov, Natalia V. Cherkashina, Andrei V. Churakov, Yan V. Zubavichus, Alexander A. Markov, Alexander E. Gekhman, Michael N. Vargaftik. // Inorganica Chimica Acta, V. 508 (2020), 119631. [3] I.P. Stolarov, N.V. Cherkashina, I.A. Yakushev, A.V. Churakov, A.B. Kornev, E.V. Fatyushina. // Russian Journal of Inorganic Chemistry, V. 65 (2020), 4, 507513 [4] Roman D. Svetogorov, Pavel V. Dorovatovskii, Vladimir A. Lazarenko. Belok/XSA diffraction beamline for studying crystalline samples at Kurchatov Synchrotron Radiation Source // Crystal Research and Technologies, V. 55 (2020), 5, 1900184

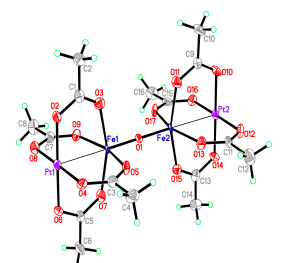

Figure 1

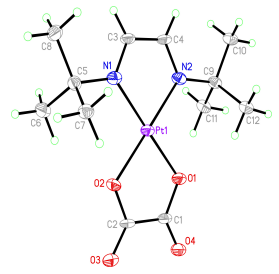

Figure 2 